\title{
Factores que determinan la mala salud mental en las personas de 16 a 64 años residentes en una gran ciudad
}

\author{
Determinants of poor mental health in people aged \\ 16 to 64 residing in a large city
}

\author{
M.M. Esteban ${ }^{1}$, M.L. Puerto ${ }^{2}$, X. Fernández Cordero ${ }^{2}$, R. Jiménez García ${ }^{3}$, \\ A. Gil de Miguel $^{3}$, V. Hernández Barrera ${ }^{3}$
}

\section{RESUMEN}

Fundamento. Conocer los factores que determinan la mala salud mental en la ciudad de Madrid.

Material y métodos. Estudio transversal con la Encuesta de Salud de la Ciudad de Madrid (ESCM'05), analizando 5.746 personas de 16 a 64 años, en los años 2004-2005. La variable dependiente fue obtenida del Cuestionario General de Salud (GHQ-12), considerando la puntuación superior a 2. Las variables independientes incluyen factores sociodemográficos, hábitos de vida, salud y medio ambiente. La calidad de vida en relación con la salud (CVRS) se midió con el cuestionario COOP / WONCA.

Resultados. La prevalencia general de mala salud mental fue del $19,2 \%$, con $\mathrm{p}<0,05$ para sexos, grupo de edad de 16 a 24 años y clases sociales menos favorecidas. Los predictores más fuertemente asociados con mala salud mental en la regresión múltiple son: mujer $\mathrm{OR}=1,48(1,23-1,78)$, menor de edad $\mathrm{OR}=3,21(2,40-4,29)$, inmigrante económico $\mathrm{OR}=1,33$ $(1,06-1,68)$, fumar o beber alcohol OR=1,22 (1,02-1,49) y 1,31 $(1,09-1,57)$, tener una enfermedad crónica $\mathrm{OR}=1,47(1,21-1,79)$, dormir menos de 8 horas $\mathrm{OR}=1,41(1,17-1,68)$, tener regular o mala percepción del estado de salud $\mathrm{OR}=1.65(1,22-2,22)$, autopercepción de mala calidad de vida OR=1,18 $(1,15-1,22)$, y limitación en las actividades sociales $\mathrm{OR}=1,34(1,07-1,69)$.

Conclusiones. Los problemas de salud mental son muy prevalentes en esta ciudad. Ser mujer, joven, inmigrante económico, tener peores estilos de vida, referir mala calidad de vida o tener limitadas las actividades sociales, son los factores que más fuerza de asociación tienen para explicar la mala salud mental.

Palabras clave. Salud mental. GHQ-12. Determinantes. Factores sociodemográficos. Factores sanitarios.

\begin{abstract}
Background. Know what factors determine the poor mental health in the city of Madrid.

Material and methods. Cross-sectional study Health Survey of the City of Madrid (ESCM'05), analyzed 5746, persons aged 16 to 64 years, in the years 2004-2005. Dependent variable was obtained from the General Health Questionnaire (GHQ-12), was considered score above 2. Independent variables included sociodemographic factors, lifestyle, health and environmental issues. The quality of life related to health (HRQOL) was measured with the questionnaire COOP / WONCA.

Results. The overall prevalence of poor mental health was $19.2 \%$, p < 0.05 for gender, age group 16 to 24 years and lower social classes. The predictors most strongly associated with poor mental health in the multiple regression are: women $\mathrm{OR}=1.48$ (1.23-1.78), minors $\mathrm{OR}=3.21 \quad(2.40$ $4.29)$, immigrant economic $\mathrm{OR}=1.33$ (1.06-1.68), smoking or drinking alcohol $\mathrm{OR}=1.22$ (1.02-1.49) and 1.31 (1.09-1.57), chronic disease $\mathrm{OR}=1.47$ (1.21-1.79), sleeping less 8 hours $\mathrm{OR}=1.41$ (1.17-1.68), having fair or poor health status perception $\mathrm{OR}=1.65(1.22-2.22)$, self-perceived poor quality of life $\mathrm{OR}=1.18$ (1.15-1.22), and limited social activities $\mathrm{OR}=1.34$ (1.07-1.69).

Conclusions. The mental health problems are highly prevalent in this city. Being woman, young, economic migrant, worse lifestyles, poor quality of life refered or limited social activities are the factors that force to explain the association with poor mental health.
\end{abstract}

Key words. Mental health. GHQ-12. Determinants. Social factors. Health factors.
1. Instituto de Salud Pública. Ayuntamiento de Madrid.

2. Departamento de Evaluación y Calidad. Unidad de Formación e Investigación. Ayuntamiento de Madrid.

3. Departamento de Medicina Preventiva y Salud Pública. Unidad de Formación e Investigación. Universidad Rey Juan Carlos.

\section{Correspondencia:}

$M^{a}$ Mercedes Esteban y Peña

Unidad de Formación e Investigación Madrid Salud C/ Juan Esplandiu, 11

28007 Madrid

E-mail: estebanpm@madrid.es

Recepción: 27 de diciembre de 2011

Aceptación provisional: 29 de febrero de 2012

Aceptación definitiva: 16 de marzo de 2012 


\section{INTRODUCCIÓN}

Los problemas de salud mental suponen una importante carga de morbilidad en todo el mundo ${ }^{1}$. Se estima que 450 millones de personas pueden padecer un trastorno mental a lo largo de su vida ${ }^{2}$. En España datos en diferentes comunidades autonómicas sitúan la prevalencia en torno al $20 \%$. La Comunidad de Madrid tenía un 23,7\% de posibles problemas de salud mental para el año 2005 y la de Cataluña del 17,6\%, con datos recogidos por el cuestionario General Health Questionnaire-12 (GHQ-12) ${ }^{3}$. Según el European Study of the Epidemiology of Mental Disorders (ESEMeD), en Europa el $11,5 \%$ de la población habría sido diagnosticada de algún problema mental en el último año ${ }^{1,2}$ y el $8,4 \%$ según ese mismo estudio en España ${ }^{4}$.

La Unión Europea, siguiendo las recomendaciones que establece la estrategia de Helsinki ${ }^{5}$, insiste en la importancia de tener en cuenta y tomar medidas sobre todos los probables factores que determinen la mala salud mental como: la desigualdad, los factores socioeconómicos y educativos, el género; así como los valores y el tiempo de ocio y relación. Los problemas de salud mental son causa de importantes pérdidas en los sistemas económicos, sociales, educativos, penales y judiciales ${ }^{1}$.

Son condicionantes de la salud mental en general y en nuestro medio en particular: los factores genéticos, biológicos, el género, las experiencias personales, familiares, sociales o económicas, así como la pobreza $^{1}$. Factores que también la determinan son: el nivel educativo, el empleo o el entorno rural o urbano ${ }^{6}$. Factores culturales y de estrés como la inmigración, los conflictos o los desastres sociales, la edad, las enfermedades físicas graves son otros agentes que establecen la salud mental de las personas. La edad aumenta claramente estos problemas. La mayor frecuencia en la población femenina es fuente de controversia sin embargo, pues si bien afecta más a las mujeres, cuando se hacen entrevistas estructuradas en el caso de la depresión, la prevalencia es si- milar según unos autores, mientras otros refieren la mayor prevalencia consolidada en mujeres para ansiedad y depresión y la menor para los trastornos psicóticos gra$\operatorname{ves}^{7,8}$.

Aunque sabemos de la importancia de los factores individuales; en el abordaje y en el tratamiento, es clave la visión del conjunto de la población, que tiene que ver con políticas para la prevención y para evitar alguna de sus consecuencias como la estigmatización.

Los trastornos mentales suponen una importante carga como enfermedad superando la de los accidentes cerebrovasculares y del resto de las enfermedades cardiovasculares, diabetes, cáncer o el Alzheimer ${ }^{9}$. En términos de calidad de vida en relación con la salud (CVRS), la depresión es la primera enfermedad en cuanto a repercusión en lo que las personas autorefieren como peor calidad de vida ${ }^{10,11}$. Las intervenciones en salud mental tienen la evidencia no sólo de mejorar el desarrollo del individuo sino de disminuir los costes económicos y sociales. En este sentido la epidemiología supone un importante instrumento de sensibilización.

Las encuestas son un instrumento diseñado por la epidemiología para conocer la prevalencia de problemas de salud en general y en particular los de salud mental en las poblaciones, bien sea como forma de aproximarse a los trastornos mentales o como manera de llegar a un diagnóstico psiquiátrico. Los avances en la epidemiología psiquiátrica han permitido introducir en entrevistas semiestructuradas diferentes instrumentos de medida a nivel poblacional como el MINI (Mini International Neuropsychiatric Interview) o el cuestionario Prime-MD (Primary Care Evaluation of Mental Disorders) entre otros. Entre los instrumentos más conocidos está el GHQ12 desarrollado por Goldberg en los años setenta del pasado siglo, como método que detecta la probabilidad de padecer un trastorno mental, pero no de un diagnóstico clínico, siendo ampliamente utilizado y validado en diversos idiomas con versiones de $60,30,20$ y 12 preguntas $^{12}$. 
El objetivo de este trabajo es conocer para nuestra ciudad cuáles son los factores que pueden determinar una mala salud mental, a través de las variables recogidas en un cuestionario general de salud.

\section{MATERIAL Y MÉTODOS}

Estudio descriptivo transversal utilizando la Encuesta de Salud de la Ciudad de Madrid (ESCM'05), con población no institucionalizada, desde noviembre de 2004 a junio de 2005. El muestreo es por conglomerado bietápico, con estratificación de unidades de primera etapa, formadas por las secciones censales y las de segunda por personas residentes en cada sección. El tamaño muestral son 8.504 entrevistas, 7.341 adultos y 1.163 menores de 16 años, con error muestral absoluto de $\pm 0,7 \%$, y una tasa inicial de respuesta del $40 \%$. La información se ha recogido por entrevista personal en el hogar a partir de un cuestionario estructurado. La población de referencia es la inscrita en el padrón municipal de habitantes a fecha de 1 de septiembre de 2004, siendo la unidad muestral los individuos, no los hogares. Más detalles de la metodología pueden encontrase en su publicación web $^{13}$.

Como variable dependiente se seleccionó la posible mala salud mental a través del cuestionario GHQ-12, que mide los problemas de salud mental a través de una escala de cuatro valores. El punto de corte se ha establecido en 3 puntos (caso > 2 puntos $)^{13,14}$. Se realizó en menores de 65 años, por lo que la población de estudio está formada por 5.746 personas. Como variables independientes se introdujeron: sociodemográficas, de hábitos y estilos de vida, de salud, y de medio ambiente.

La clase social fue recogida según la Clasificación Nacional de Ocupación (CNO), recomendada por la Sociedad Española de Epidemiología. La clase I y II corresponden a directivos y universitarios; la clase III a administrativos y personas que apoyan la gestión, así como a trabajadores por cuenta propia; la clase IV a trabajadores cualificados, la clase $\mathrm{V}$ a trabajadores no cualificados. La clase $\mathrm{X}$ corresponde a un grupo sin clasificar por ausencia de $\mathrm{CNO}$, cuyo perfil es el de mujer, jubilado/pensionista, amas de casa, que nunca han trabajado y que son en la actualidad los sustentadores principales del hogaris.

Con la variable de distritos se han hecho cuatro grupos usando conglomerados. Para ello se ha considerando la esperanza media de vida al nacer (EMVN), convertida en un índice de 0 a 1 , los niveles de renta con la Renta Bruta Disponible per cápita (RBDpc) haciendo un índice en el que se considera 100 la renta de la ciudad y por último el grado escolar, como proporción de personas con grado escolar, estableciendo un índice porcentual. Las agrupaciones se hicieron con el programa estadístico SPSS siendo el Grupo 1 el más desfavorecido y el Grupo 4 el menos ${ }^{3,13}$.

Por inmigrante se han considerado los económicos, como personas procedentes de los países de Europa del Este, África, América del Sur y Asia (excepto Japón). Por parado se incluye a toda persona entre 45-64 años que se encuentra en situación de paro laboral.

Para la CVRS se ha empleado el cuestionario COOP/WONCA, validado en español con escala tipo Likert del 1 al 5 , que mide de forma inversa la calidad de vida ${ }^{16,17}$.

El índice de masa corporal (IMC) se calculó con el valor que decían que median y pesaban, considerándose obesos por encima de $30 \mathrm{~kg} / \mathrm{m}^{2}$. El consumo de alcohol se ha extraído de la pregunta de la ESCM'05: ¿En los últimos 12 meses ha consumido usted alguna bebida alcohólica con una frecuencia de al menos una vez al mes? No ejercicio físico significa que no hace ninguna actividad física en su tiempo libre.

Las variables de: autopercepción del estado de salud, apoyo social, actividades sociales, sentimientos, disponibilidad de horas libres al día, vivienda, autopercepción del medio ambiente y ruido se recogieron con las preguntas literales del cuestionario de la Encuesta de Salud de la ciudad de Madrid (ESCM'05) como se puede ver en la tabla 1. 
Tabla 1. Variables y preguntas empleadas de la ESCM'05. Encuesta de Salud de la Ciudad de Madrid 2004-2005

\begin{tabular}{|c|c|}
\hline Variables & Pregunta realizada \\
\hline Autopercepción del estado de salud & $\begin{array}{l}\text { ¿Cómo considera que es su estado de salud? } \\
\text { Se agrupan en tres categorías: excelente-muy buena, buena, mala- } \\
\text { regular }\end{array}$ \\
\hline Apoyo social & ¿Había alguien dispuesto a ayudarle si hubiera necesitado ayuda? \\
\hline Actividades sociales & $\begin{array}{l}\text { ¿Su salud física y estado emocional han limitado sus actividades } \\
\text { sociales con la familia, amigos, vecinos o grupos? }\end{array}$ \\
\hline Sentimientos & $\begin{array}{l}\text { ¿En qué medida le han molestado los problemas emocionales tales } \\
\text { como sentimientos de ansiedad, depresión, irritabilidad o tristeza } \\
\text { y desánimo? }\end{array}$ \\
\hline Disponibilidad de horas libres al día & $\begin{array}{l}\text { ¿Cuántas horas libres dispone al día, excluyendo los fines de } \\
\text { semana? }\end{array}$ \\
\hline Vivienda & ¿Cuántos $\mathrm{m}^{2}$ útiles tiene aproximadamente la vivienda? \\
\hline La autopercepción del medio ambiente: & $\begin{array}{l}\text { ¿Cómo considera que es la calidad del medio ambiente de su barrio? } \\
\text { Se agrupan en tres categorías: excelente-buena, regular, y mala-muy } \\
\text { mala }\end{array}$ \\
\hline El ruido & $\begin{array}{l}\text { ¿Considera que el ruido procedente del exterior de su vivienda le } \\
\text { resulta molesto? }\end{array}$ \\
\hline
\end{tabular}

\section{Análisis estadístico}

Se ha realizado un análisis descriptivo de la población de estudio, usando medias y porcentajes, con desviación estándar (DE), e intervalos de confianza (IC al 95\%). Para la comparación bivariante de proporciones se empleó el test estadístico $\chi^{2}$.

Para la relación entre la variable dependiente y las variables independientes se utilizó el análisis de regresión logística simple o binaria y múltiple, considerando como variable dependiente la mala salud mental y como variables independientes las sociodemográficas, de hábitos y estilos de vida, de salud y medioambientales. Los efectos sobre la variable dependiente se han valorado con la Odds Ratio, con intervalo de confianza.

Se han calculado coeficientes de ponderación por individuo y por distrito teniendo en cuenta el diseño muestral de afija- ción no proporcional por distritos. Antes del análisis de la muestra se determinó la variación de los resultados muestrales con IC al $95 \%$, para medias y proporciones

El análisis estadístico se ha realizado con el programa SPSS 16.0 y el Stata 11. Este análisis se ha llevado a cabo en bases de datos anonimizadas de uso público, por lo que no es necesario contar con la aprobación de un comité de ética de acuerdo a la legislación española.

\section{RESULTADOS}

En la tabla 2 se muestran las características sociodemográficas en cuanto a edad, sexo, nacionalidad grupos de edad y clase social de la muestra sobre la que se realiza el estudio ( $n=5.746$ personas) y las de la población de más de 16 años de la encuesta. 
Tabla 2. Prevalencia de GHQ-12 > 2 puntos en las personas de 16-64 años residentes en la Ciudad de Madrid según variables sociodemográficas

\begin{tabular}{|c|c|c|c|c|c|c|}
\hline \multirow[b]{2}{*}{ Variables } & \multicolumn{3}{|c|}{$\begin{array}{l}\text { Población } \\
\text { de estudio }\end{array}$} & \multicolumn{3}{|c|}{$\begin{array}{l}\text { Población total de la encuesta } \\
>16 \text { años }\end{array}$} \\
\hline & $\mathrm{N}$ & Porcentaje & (IC 95\%) & $\mathrm{N}$ & Porcentaje & (IC 95\%) \\
\hline Entrevistados & 5.746 & & & 7.341 & & \\
\hline \multicolumn{7}{|l|}{ Sexo } \\
\hline Hombre & 2.777 & $48,3 \%$ & $(46,4-50,2)$ & 3.396 & $46,3 \%$ & $(44,6-48,0)$ \\
\hline Mujer & 2.968 & $51,7 \%$ & $(49,9-53,5)$ & 3.945 & $53,7 \%$ & $(52,1-55,3)$ \\
\hline \multicolumn{7}{|l|}{ Nacionalidad } \\
\hline Española & 4.813 & $83,8 \%$ & $(82,8-84,8)$ & 6.375 & $86,8 \%$ & $(86,2-87,8)$ \\
\hline Extranjera & 809 & $14,1 \%$ & $(11,7-16,5)$ & 836 & 11,4 & $(9,2-13,6)$ \\
\hline Ambas & 108 & $1,9 \%$ & $(-0,7-4,5)$ & 115 & 1,6 & $(-0,7-3,9)$ \\
\hline \multicolumn{7}{|l|}{ Grupos de edad } \\
\hline De 16 a 24 años & 870 & $15,1 \%$ & $(12,7-17,5$ & 870 & 11,8 & $(9,7-3,9)$ \\
\hline De 25 a 44 años & 2.952 & $51,4 \%$ & $(49,6-53,2)$ & 2.953 & 40,2 & $(38,4-42,0)$ \\
\hline De 45 a 64 años & 1.923 & $33,5 \%$ & $(31,4-35,6)$ & 1.923 & 26,2 & $(24,2-28,2)$ \\
\hline Más de 65 años & - & - & & 1.595 & 21,7 & $(19,7-23,7)$ \\
\hline \multicolumn{7}{|l|}{ Clase social } \\
\hline Clases I-II & 1.872 & $32,6 \%$ & $(30,5-34,7)$ & 2.203 & $30 \%$ & $(28,1-31,9)$ \\
\hline Clase III & 1.371 & $23,9 \%$ & $(21,6-26,2)$ & 1.719 & $23,4 \%$ & $(21,4-25,4)$ \\
\hline Clase IV & 1.758 & $30,6 \%$ & $(28,4-32,8)$ & 2.302 & $31,4 \%$ & $(29,5-33,3)$ \\
\hline Clase V & 613 & $10,7 \%$ & $(8,3-13,1)$ & 778 & $10,6 \%$ & $(8,4-12,8)$ \\
\hline Clase X & 80 & $1,4 \%$ & $(-1,2-4,0)$ & 280 & $3,8 \%$ & $(1,6-6,0)$ \\
\hline
\end{tabular}

$\mathrm{N}=$ Número de casos; IC $95 \%=$ Intervalo de confianza para 95\% de seguridad.

En la tablas 3 y 4 se muestran la puntuación $>2$ del GHQ-12, según variables sociodemográficas, de hábitos y estilos de vida, de salud y medioambientales y las Odds Ratio con los intervalos de confianza, para identificar los predictores de la mala salud mental en la regresión logística simple o binaria realizada. La prevalencia para toda la población menor de 65 años se sitúa en el 19,2\% de las personas, siendo el $23,4 \%$ en las mujeres y en el 14,6\% de los hombres, con una $\mathrm{p}<0,05$ según el análisis bivariado (test de $\chi^{2}$ ). Por grupos de edad, es el $20,3 \%$ en el grupo de 16 a 24 años con significación estadística ( $\mathrm{p}>$ de 0,05 ). En las diferentes clases sociales se observan prevalencias de $15,7 \%, 17,2 \%$, $22,4 \%, 24 \%$ y $32,1 \%$ respectivamente para los grupos sociales de Clase I-II, III, IV, V y X con $\mathrm{p}<0,05$. Para el Grupo 1 de distritos, correspondiente a los distritos de Usera, Vallecas y Villaverde la prevalencia es del $25,7 \%$, siendo significativa la diferencia por esta asociación en el análisis bivariado. En los grupos socia- les de parados, e inmigrantes las prevalencias son del $32,9 \%$ y del $21,9 \%$, siendo en los primeros la $\mathrm{p}>0,05$. Aquellas personas con alguna enfermedad crónica tienen una prevalencia del $28 \%$ con $\mathrm{p}<0,05$. Las personas que autoperciben regular y mala salud, las que perciben mal y muy mal medio ambiente, y las que limitan sus actividades sociales, tienen respectivamente el 45,8\%, 33,7\%,y $46,5 \%$ de GHQ-12 > de 2 . Como predictores positivos, con significación estadística $\mathrm{p}<$ 0,05 de la mala salud mental han resultado en la regresión logística simple o binaria el sexo (femenino), la clase social (IV, V y X), pertenencia al Grupo 1 de los distritos, el nivel de estudios (secundarios y primarios), el estado civil (separados, divorciados, viudos), la condición de inmigrante económico, la no realización de ejercicio físico, el fumar, el tener una enfermedad crónica, las horas de sueño, la mala CVRS, la mala percepción del medio ambiente, el tamaño de la vivienda y las horas libres disponibles. 
Tabla 3. Prevalencia de GHQ-12 > 2 puntos en las personas de 15-64 años residentes en la Ciudad de Madrid según variables sociodemográficas

\begin{tabular}{|c|c|c|c|c|}
\hline & $\mathbf{N}$ & $\begin{array}{l}\text { GHQ-12 > } 2 \\
\text { Porcentaje }\end{array}$ & OR (IC 95\%) & Sig. \\
\hline Total de personas estudiadas & 5.746 & & & \\
\hline \multicolumn{5}{|l|}{ Sexo } \\
\hline Hombres & 396 & $14,6 \%$ & 1 & \\
\hline Mujeres* & 684 & $23,4 \%$ & $1,85(1,61-2,12)$ & 0,000 \\
\hline Total & 1.080 & $19,2 \%$ & & \\
\hline \multicolumn{5}{|l|}{ Grupos de edad } \\
\hline De 16 a 24 años & 173 & $20,3 \%$ & 1 & 0,276 \\
\hline De 25 a 44 años & 532 & $18,4 \%$ & $0,88(0,72-1,07)$ & 0,194 \\
\hline De 45 a 64 años & 376 & $20,0 \%$ & $0,97(0,79-1,19)$ & 0,773 \\
\hline \multicolumn{5}{|l|}{ Clase social } \\
\hline Clases I-II & 288 & $15,7 \%$ & 1 & 0,000 \\
\hline Clases III & 231 & $17,2 \%$ & $1,09(0,90-1,32)$ & 0,373 \\
\hline Clase IV* & 383 & $22,4 \%$ & $1,61(1,36-1,91)$ & 0,000 \\
\hline Clase $V^{*}$ & 143 & $24,0 \%$ & $1,68(1,34-2,11)$ & 0,000 \\
\hline Clase $X^{*}$ & 25 & $32,1 \%$ & $2,51(1,53-4,11)$ & 0,000 \\
\hline \multicolumn{5}{|l|}{ Distritos } \\
\hline Grupo 4 & 194 & $16,1 \%$ & 1 & 0,000 \\
\hline Grupo $1^{*}$ & 228 & $25,7 \%$ & $1,61(1,29-2,00)$ & 0,000 \\
\hline Grupo 2 & 240 & $17,4 \%$ & $1,02(0,83-1,26)$ & 0,832 \\
\hline Grupo 3 & 419 & $19,4 \%$ & $1,20(1,00-1,45)$ & 0,054 \\
\hline \multicolumn{5}{|l|}{ Nivel de estudios } \\
\hline Terciarios & 280 & $15,3 \%$ & 1 & 0,000 \\
\hline Primarios* & 187 & $24,5 \%$ & $1,66(1,34-2,05)$ & 0,000 \\
\hline Secundarios* & 611 & $20,2 \%$ & $1,33(1,13-1,56)$ & 0,000 \\
\hline \multicolumn{5}{|l|}{ Estado civil } \\
\hline Casado/pareja & 582 & $18,5 \%$ & 1 & 0,000 \\
\hline Soltero/a & 400 & $18,6 \%$ & $1,00(0,87-1,15)$ & 0,929 \\
\hline Separado/a* & 43 & $33,9 \%$ & $2,28(1,56-3,33)$ & 0,000 \\
\hline Divorciado/a* & 30 & $33,3 \%$ & $2,17(1,38-3,41)$ & 0,001 \\
\hline Viudo/a* & 26 & $30,2 \%$ & $1,89(1,18-3,03)$ & 0,008 \\
\hline \multicolumn{5}{|l|}{ Grupos sociales } \\
\hline \multicolumn{5}{|l|}{ Personas que viven solas } \\
\hline No & & & 1 & \\
\hline Sí & 55 & $18,1 \%$ & $0,92(0,68-1,25)$ & 0,621 \\
\hline \multicolumn{5}{|l|}{ Parados } \\
\hline No & & & 1 & \\
\hline Sí & 132 & $32,8 \%$ & $0,72(0,43-1,19)$ & 0,203 \\
\hline \multicolumn{5}{|l|}{ Inmigrantes Económicos } \\
\hline No & & & 1 & \\
\hline $\mathrm{Sí}^{*}$ & 198 & $21,9 \%$ & $3,21(1,43-7,20)$ & 0,005 \\
\hline
\end{tabular}

${ }^{*} \mathrm{p}<0,05$ estadísticamente significativa para la diferencia entre los grupos con la prueba de $\chi 2$;

$\mathrm{N}=$ Número de casos; OR Odds Ratio; IC: intervalo de confianza al 95,0\% 
Tabla 4. Prevalencia de GHQ-12 > 2 puntos en las personas de 16-64 años residentes en la ciudad de Madrid según hábitos de estilos de vida, de salud y medioambientales

\begin{tabular}{|c|c|c|c|c|}
\hline & $\mathbf{N}$ & $\begin{array}{l}\text { GHQ-12 > } 2 \\
\text { Porcentaje }\end{array}$ & OR (IC 95\%) & Sig. \\
\hline \multicolumn{5}{|l|}{ Realización de ejercicio físico } \\
\hline Sí & & & 1 & \\
\hline $\mathrm{No}^{*}$ & 429 & $24,2 \%$ & $1,52(1,32-1,75)$ & 0,000 \\
\hline \multicolumn{5}{|l|}{ Tabaco } \\
\hline No fumador & 507 & $16,9 \%$ & 1 & 0,000 \\
\hline Exfumador * & 172 & $21,1 \%$ & $1,39(1,14-1,69)$ & 0,000 \\
\hline Fumador* & 401 & $22,3 \%$ & $1,49(1,28-1,73)$ & 0,000 \\
\hline \multicolumn{5}{|l|}{ Alcohol } \\
\hline No & & & 1 & \\
\hline Sí & 622 & $19,6 \%$ & $1,04(0,90-1,19)$ & 0,570 \\
\hline \multicolumn{5}{|l|}{ Presencia de enfermedad crónica } \\
\hline No & & & 1 & \\
\hline $\mathrm{Si}^{*}$ & 744 & $28,2 \%$ & $3,01(2,60-3,47)$ & 0,000 \\
\hline IMC & & & $0,98(0,97-1,00)$ & 0,133 \\
\hline Horas de sueño * & & & $0,86(0,81-0,91)$ & 0,000 \\
\hline \multicolumn{5}{|l|}{ Autopercepción del Estado de Salud } \\
\hline Excelente-Muy buena & 203 & $10,1 \%$ & 1 & 0,062 \\
\hline Buena & 464 & $17,1 \%$ & $0,93(0,76-1,13)$ & 0,470 \\
\hline Mala-Regular & 408 & $45,8 \%$ & $1,18(0,91-1,53)$ & 0,189 \\
\hline Calidad de vida * & & & $1,28(1,25-1,31)$ & 0,000 \\
\hline \multicolumn{5}{|l|}{ Percepción de medio ambiente } \\
\hline Excelente-buena & 590 & $16,8 \%$ & 1 & 0,000 \\
\hline Regular* & 382 & $22,0 \%$ & $1,27(1,09-1,48)$ & 0,002 \\
\hline Mala-muy mala* & 97 & $33,7 \%$ & $2,09(1,58-2,77)$ & 0,000 \\
\hline \multicolumn{5}{|l|}{ Ruido } \\
\hline No & & & 1 & \\
\hline Sí & 523 & $21,1 \%$ & $0,87(0,74-1,01)$ & 0,069 \\
\hline Vivienda* & & & $0,99(0,99-1,00)$ & 0,044 \\
\hline \multicolumn{5}{|l|}{ Apoyo social } \\
\hline Sí & & & 1 & \\
\hline $\mathrm{No}^{*}$ & 59 & $31,6 \%$ & $0,65(0,45-0,93)$ & 0,021 \\
\hline \multicolumn{5}{|l|}{ Limitación de actividades sociales } \\
\hline No & & & 1 & \\
\hline $\mathrm{Si}^{*}$ & 532 & $46,5 \%$ & $0,32(0,27-0,38)$ & 0,000 \\
\hline \multicolumn{5}{|l|}{ Sentimientos } \\
\hline No le han molestado & & & 1 & \\
\hline Sí le han molestado* & 770 & $41,4 \%$ & $0,19(0,16-0,22)$ & 0,000 \\
\hline Horas libres de que dispone al día* & & & $1,04(1,02-1,06)$ & 0,000 \\
\hline
\end{tabular}

${ }^{*} \mathrm{p}<0,05$ estadísticamente significativa para la diferencia entre los grupos con la prueba de $\chi 2$;

$\mathrm{N}=$ Número de casos; OR Odds Ratio; IC Intervalo de Confianza al 95\%. 
En la tabla 5 se muestran los resultados tras introducir todas las variables en la regresión logística múltiple y que presentan un OR por encima de 1 , es decir con un ventaja positiva, teniendo de éstas, significación estadística: el sexo (femenino), la edad (joven), la condición de inmigran- te económico, el ser fumador o bebedor, el tener una enfermedad crónica, dormir menos de 8 horas al día, percibir regular o mal estado de salud, autopercibir una mala calidad de vida, estar limitado en actividades sociales y la presencia de sentimientos molestos.

Tabla 5. Variables sociodemográficas, de hábitos y estilos de vida, de salud y medioambientales en las personas de 16-64 años residentes en la Ciudad de Madrid asociada con a la salud mental. Resultados del modelo de regresión logística múltiple

\begin{tabular}{|c|c|c|c|c|}
\hline Variables & & OR & IC95\% & Sig. \\
\hline \multirow{2}{*}{ Sexo } & Hombre & 1 & & \\
\hline & Mujer * & 1,48 & $(1,23-1,78)$ & 0,000 \\
\hline \multirow{3}{*}{ Edad } & 45-64 años & 1 & & \\
\hline & 25-44 años* & 1,57 & $(1,28-1,92)$ & 0,000 \\
\hline & 16-24 años* & 3,21 & $(2,40-4,29)$ & 0,000 \\
\hline \multirow{2}{*}{ Inmigrante económico } & No & 1 & & \\
\hline & $\mathrm{Si}^{*}$ & 1,33 & $(1,06-1,68)$ & 0,014 \\
\hline \multirow{3}{*}{ Tabaco } & No fumador & 1 & & \\
\hline & Ex fumador & 1,24 & $(0,96-1,60)$ & 0,098 \\
\hline & Fumador* & 1,22 & $(1,02-1,49)$ & 0,047 \\
\hline \multirow{2}{*}{ Alcohol } & No & 1 & & \\
\hline & $\mathrm{Si}^{*}$ & 1,31 & $(1,09-1,57)$ & 0,004 \\
\hline \multirow{2}{*}{$\begin{array}{l}\text { Presencia de enfermedad } \\
\text { crónica }\end{array}$} & No & 1 & & \\
\hline & $\mathrm{Si}^{*}$ & 1,47 & $(1,21-1,79)$ & 0,000 \\
\hline \multirow{2}{*}{ Horas de sueño } & 8 o más horas & 1 & & \\
\hline & Menos de $8^{*}$ & 1,41 & $(1,17-1,68)$ & 0,000 \\
\hline \multirow{3}{*}{$\begin{array}{l}\text { Autopercepción del Estado de } \\
\text { Salud }\end{array}$} & Excelente-muy buena & 1 & & \\
\hline & Buena & 1,05 & $(0,84-1,31)$ & 0,666 \\
\hline & Regular-mala* & 1,65 & $(1,22-2,22)$ & 0,001 \\
\hline Calidad de vida & Wonca $^{*}$ & 1,18 & $(1,15-1,22)$ & 0,000 \\
\hline \multirow{2}{*}{$\begin{array}{l}\text { Limitación de actividades } \\
\text { sociales }\end{array}$} & No & 1 & & \\
\hline & $\mathrm{Si}^{*}$ & 1,34 & $(1,07-1,69)$ & 0,010 \\
\hline \multirow{2}{*}{ Sentimientos } & No le han molestado & 1 & & \\
\hline & Sí le han molestado* & 2,86 & $(2,35-3,49)$ & 0,000 \\
\hline
\end{tabular}

*Diferencia significativa; OR Odds Ratio; Sig: significación estadística, valor de p< 0,05; IC 95\% Intervalo de confianza al $95,0 \%$. 


\section{DISCUSIÓN}

Este estudio de factores que determinan la mala salud mental en la ciudad de Madrid revela la importancia que tiene el problema de la salud mental en la ciudad, ya que una de cada cinco personas tiene la posibilidad de tener un posible trastorno, como ocurre en el resto del territorio nacional. Asimismo se evidencia la concordancia de los factores asociados con los apuntados en otros estudios comunitarios respecto de variables sociodemográficas, de hábitos y estilos de vida, de salud y también de algunas variables medioambientales $^{1,4,5}$.

Considerando el primer grupo de factores: el ser mujer es una variable claramente referenciada y asociada a la posibilidad de tener un problema de salud mental ${ }^{8}$. La condición laboral o los roles sociales son apuntados en diferentes estudios, más que la propia condición femenina; así como tener menos recursos económicos, menor autonomía y mayor dependencia del hombre e incluso combinar el trabajo fuera de casa con las tareas domésticas y el cuidado de los hijos. Las fuentes disponibles sobre la salud mental de hombres y mujeres refieren que las mujeres tienen un perfil más desfavorable, y las variables relacionadas son debido a los roles sociales asociados con el sexo. De los trabajos que han analizado las variables que en los diferentes sexos explican la mala salud mental, es el estatus laboral, el que está significativamente relacionado con la mala salud mental ${ }^{17,18}$. La edad está claramente relacionada con los problemas de salud mental, como ocurre en los grupos de más edad. En nuestro trabajo existe una asociación entre los grupos más jóvenes, como lo referencian otros autores y la posibilidad de tener un trastorno mental (no se incluyen los mayores de 65 años), hecho que indudablemente tiene mucha relación con la problemática asociada a la adolescencia y periadolescencia ${ }^{19}$. La clase social y el nivel educativo son asimismo variables que explican la peor salud mental, al igual que la pertenencia a un determinado distrito, en este caso los más desfavorecidos. Estas variables pierden asociación en el análisis múltiple, por la menor fuerza de agrupación ante la presencia de las otras variables ${ }^{17,20}$, lo que en este caso obviaría las limitaciones en la validez de la escala de la clase social al perderse la de distrito y educación también. El ocio elegido y organizado es un factor reseñado en la prevención y en el tratamiento de los problemas de salud mental. El ocio se define como parámetro de salud, un nuevo paradigma de salud y el tiempo libre una parte de la salud integral. Esta es una de las variables que se mantiene en el análisis multivariante ${ }^{21}$. El paro laboral, no es ni variable explicativa ni justifica el riesgo de padecer mala salud mental en nuestro estudio. Esto debe ser motivo de futuros análisis ya que la representación en esta muestra es escasa, y el paro laboral en la Ciudad de Madrid se ha duplicado desde el 2005 al $2009^{22}$. Respecto los inmigrantes económicos, se evidencian como un factor de riesgo de mala salud mental. Referencias bibliográficas concluyen que la inmigración "per se" no produciría un incremento del riesgo de padecer enfermedades mentales sino que dependería, de experiencias traumáticas del proceso migratorio. Procesos de adaptación a la cultura del país anfitrión, condiciones de la vivienda, falta de apoyo social, ingresos precarios, así como dificultades ambientales son aceptados como causa de la emergencia de patologías mentales en este colectivo ${ }^{23}$.

Abordando los hábitos y estilos de vida: la bondad de la realización de ejercicio físico regular, está referenciada tanto para el mantenimiento de las facultades físicas, y la prevención de la dependencia, como para mejorar las capacidades sociales, y evitar así los problemas de ansiedad o depresión. La no realización de ejercicio físico es un factor que claramente explica la mayor posibilidad de tener un problema de salud mental en el análisis binario ${ }^{24}$. Hay mayores tasas de fumadores entre el las personas con problemas de salud mental. Entre un 30 y $60 \%$ de personas con problemas mentales fuman según diversos estu$\operatorname{dios}^{25}$. En nuestro trabajo vemos el reflejo de esta mayor prevalencia conocida, al explicar con mayor posibilidad el tener un 
problema de salud mental las personas que fuman, variable que se mantiene cuando se incorporan las sociodemograficas y de salud. En el alcoholismo crónico es referenciada la asociación con la mayor morbilidad psiquiátrica. Hasta en un $37 \%$ hay presencia de enfermedades mentales según el estudio ECA (Results From the Epidemiologic Catchment Area Study). Se mantiene la posible mala salud mental, con la variable de consumo de alcohol en el análisis multivariante, con el resto de las variables sociales y de salud ${ }^{26}$.

Con respecto a los aspectos de salud: las enfermedades crónicas son causa importante de pérdida de salud, además de generar dependencia física y pérdida de calidad de vida. Algunas se asocian a la mayor presencia de depresión o de problemas de ansiedad como son las enfermedades cardiacas, las neurológicas o la diabetes. Es de los factores incorporados el que tiene más ventaja para explicar la presencia de una posible enfermedad mental ${ }^{27,28}$, además de mantenerse con la incorporación del resto de las variables sociodemográficas. El sueño es un factor que claramente se muestra como protector frente a los posibles problemas de salud mental. Diversos estudios referencian la importancia de las horas de sueño para evitar problemas de salud mental en general ${ }^{6}$. La autopercepción del estado de salud se comporta como explicativo de la mala salud mental, en el análisis multivariante, variable muy relacionada con la propia salud de las personas y con una gran correlación con la calidad de vida autopercibida $^{28}$. La CVRS tiene una razón de ventajas positiva y es un factor claramente explicativo de la mala salud mental en los ciudadanos de Madrid, además de comportarse como el más relevante en este sentido ya que por cada punto que aumenta en el cuestionario la OR es de 1,18 . La depresión es de las enfermedades crónicas la que más explica la mala calidad de vida de las personas, según diversos estudios. Resulta de gran interés que se confirme como un factor determinante de la mala salud mental y sea tan explicativo de la misma ${ }^{15}$.

El ruido, que es un importante problema ambiental y resulta una de las quejas más frecuentes dentro de las grandes urbes, no se constata en este estudio con una relación significativa entre la apreciación subjetiva del ruido del exterior y la salud mental. Aunque la exposición a elevados niveles de ruido se ha asociado con el desarrollo de algunos trastornos mentales, la relación entre el ruido ambiental y los efectos en la salud mental no son concluyentes, aunque cabe presumir que pueda influir acelerando o intensificando su desarrollo al actuar como factor estresante $^{29}$. Respecto del hacinamiento, numerosos trabajos evidencian su relación con la salud mental y con el diseño de la vivienda o la calidad habitacional. En nuestro estudio el tamaño de la vivienda per se resulta débilmente predictor de la mala salud mental ${ }^{30}$. La mala percepción del medio ambiente destaca con importante ventaja como uno de los valores predictores de mala salud mental con respecto a aquellos que consideran que la calidad del medio ambiente de su barrio es buena. Las condiciones precarias que determinan el medio ambiente, ligadas estrechamente a barrios económicamente deprimidos, como la mala iluminación, la contaminación ambiental, la falta de limpieza, ausencias de zonas verdes etc. se apuntan como causantes en este sentido ${ }^{3}$.

Las fortalezas de nuestro estudio se basan en la amplia muestra, el gran número y generalidad de posibles factores determinantes, y el análisis multivariado.

Algunas limitaciones que presenta el trabajo son:

El uso de datos autorreferidos, en cuanto a determinación de la mala salud mental. Por este motivo la información puede estar sesgada por la tendencia de los individuos para dar respuestas socialmente deseables en las entrevistas. Sin embargo, en este sentido hay que citar la concordancia con otros estudios en este campo, lo que apoya la utilidad y representatividad de la muestra.

La ESCM'O5 no incluye personas institucionalizadas, por lo que puede haber un sesgo, al no incluirse hogares de ancianos $\mathrm{y}$ residencias, con alta prevalencia de problemas de salud mental. 
La población de más de 65 años no se ha incluido en el estudio, porque el cuestionario GHQ-12 podría tener muchos falsos positivos en esta población, lo cual limita la extrapolación a todos los grupos etarios adultos ${ }^{3,13}$.

El trabajo se ha realizado en un entorno urbano, cuya prevalencia de problemas de salud mental es mayor que en entornos rurales, por lo que la validez de los resultados para la población general en este sentido debe ser considerada con precaución.

Por último la tasa inicial de respuesta fue del $40 \%$ y de la no respuesta ligeramente superior entre las mujeres, los de menor nivel educativo y los inmigrantes, por lo que en este aspecto se debe de considerar un posible sesgo ${ }^{13}$.

A modo de conclusiones: de los resultados de nuestro estudio, que provienen de una amplia muestra de la ciudad de Madrid, se puede decir que los problemas de salud mental tienen una importante prevalencia en esta ciudad. El ser mujer, joven, inmigrante económico, fumar, beber, tener una enfermedad crónica, dormir menos de 8 horas, referir mal estado de salud, referir mala calidad de vida o limitarse en las actividades sociales son los factores que más fuerza de asociación tienen para explicar la mala salud mental. Por ello podemos plantear recomendaciones en nuestro entorno inmediato dando a conocer los resultados a las autoridades públicas para que emprendan acciones y planifiquen en el ámbito medioambiental, social o sanitario, que permitan proponer e implementar estrategias de salud pública, de prevención, promoción y educación para reducir la prevalencia y la morbilidad de estos problemas de salud mental en los madrileños, además de extrapolar los resultados a otros entornos urbanos.

\section{Agradecimientos}

Los autores agradecen al Instituto de Salud Pública (Madrid Salud-Ayuntamiento de Madrid) el acceso a las bases de datos de la Encuesta de salud de la Ciudad de Madrid 2005.

\section{BIBLIOGRAFÍA}

1. Comisión de las Comunidades Europeas. Mejorar La Salud Mental de la Población. Hacia Una Estrategia de la Unión Europea en Materia de Salud Mental. 2005. Disponible en http://eur-lex.europa.eu/LexUriServ/site/es/ com/2005/com2005_0484es01.pdf. Consultado en octubre 2011 .

2. Alonso J, Angermeyer MC, Bernert S, Bruffaerts R, BRugha TS, Bryson H et al. Prevalence of mental disorders in Europe: results from the European Study of the Epidemiology of Mental Disorders (ESEMeD) project. Acta Psychiatr Scand 2004; Suppl. 420: 21-27.

3. Madrid Salud. Estudio de Salud de la Ciudad de Madrid 2006. Ayuntamiento de Madrid. Disponible en: http://www.madridsalud.es. Consultado el 20/03/2011.

4. Haro JM, Palacín C, Vilagut G, Martínez M, Bernal M, Luque I, Codony M, Dolz M, Alonso J, Grupo ESEMeD-España. Prevalencia de los trastornos mentales y factores asociados: resultados del estudio ESEMeD-España. Med Clin (Barc) 2006; 126: 445-451.

5. World Health Organisation. Mental Health: Facing the Challenges, Building Solutions: report from the WHO European Ministerial Conference of Helsinki. Copenhagen: WHO Regional Office for Europe. 2005.

6. The European Opinion Research Group (EORG). The Mental Health Status of the European Population. Eurobarometer 58.2. disponible en: http: //ec.europa.eu/health / ph_determinants/life_style/mental_eurobaro.pdf. Consultado Octubre 2011.

7. Montero I, Aparicio D, Gómez-Beneyto M, Moreno-Küstner B, Reneses B, Usall J et al. Género y salud mental en un mundo cambiante. Gac Sanit 2004; 18: 75-181.

8. SÁnchez-López MP, López-García JJ, Dresch V, Corbalán J. Sociodemographic, psychological and health-related factors associated with poor mental health in Spanish women and men in midlife. Women Health 2008; 48: 445-465.

9. García-Altés A, Pinilla J, Peiró S. Aproximación a los pesos de calidad de vida de los "años de vida ajustados por calidad" mediante el estado de salud autopercibido. Gac Sanit 2006; 20: 457-464.

10. Kessler RC, Berglund P, Demler O, Jin R, Koretz D, MeriKangas KR et al. The epidemiology of major depressive disorder: results from the National Comorbidity Survey Replication (NCS-R). JAMA 2003; 89: 3095-3105. 
11. Ricci-Cabello I, Ruiz-Pérez I, Plazaola-Castaño J, Montero PIÑar I. Morbilidad psíquica, existencia de diagnóstico y consumo de psicofármacos, diferencias por comunidades autónomas según la encuesta nacional de salud de 2006. Rev Esp Salud Pública 2010; 84: 29-41.

12. Baca Baldomero E, Sáiz Ruiz J, Porras Chavarino A. Detección de trastornos mentales por médicos no psiquiatras:utilidad del cuestionario PRIME-MD. Med Clin 2001; 116: 504-509.

13. Madrid City Council. Madrid City Health Survey (Encuesta de Salud de la ciudad de Madrid, ESCM05). Disponible en http://www. madridsalud.es/publicaciones/OtrasPublicaciones/encuesta-salud2005.pdf. Consultado en enero 2012.

14. SÁnchez-López MP, Dresch V. The 12-Item general health questionnaire (GHQ-12): reliability, external validity and factor structure in the Spanish population. Psicothema 2008; 20: 839-843.

15. REGIDOR E. The Goldthorpe social class classification: reference frame-work for a proposal for the measurement of social class by the Working Group of the Spanish Society of Epidemiology. Rev Esp Salud Pública 2001; 75: 13-22.

16. WONCA, ERGHO. Measuring functional health status with the COOP/ WONCA charts. Available at: http://www.globalfamilydoctor.com/publications/publications. asp; 1995. Consultado en junio 2011.

17. LIZÁn L, ReIg A. Cross cultural adaptation of a health related quality of life measurement: the Spanish version of the COOP/WONCA cartoons. Aten Primaria 1999; 24: 75-82.

18. Sánchez López MP, Aparicio García ME, Dresch V. Ansiedad, autoestima y satisfacción autopercibida como predictores de la salud: diferencias entre hombres y mujeres. Psicothema 2006; 18: 584-590.

19. Organización Mundial de la Salud. El contexto de la salud mental. Conjunto de Guías sobre Servicios y Políticas de Salud Mental. OMS Editores Medicos S.A EDINSA. 2005.
20. Benach J, Amable M. Las clases sociales y la pobreza. Gac Sanit 2004; 18: 16-23.

21. ADOZ Boletín del centro de documentación en Ocio. Universidad de Deusto 2002, nº 22 .

22. Garrido RP, Castillo H, Benavides FG, Ruiz P. Situación laboral y salud mental. Resultados de un estudio transversal. Gac Sanit 1994; 8: 128-132.

23. Achotegui Loizte J, Morales Suárez-Varela MM, Cervera Boada P, Quirós Bauset C, Vicente Pérez Fuster J, Gimeno Clemente N et al. Revisión sistemática sobre la prevalencia de patologías mentales en inmigrantes en España. Norte de Salud Mental. 2009; 35; 50-55.

24. Leibovich P, IANCU I. Physical exercise among psychiatric patients: physical and mental effects. Harefuah 2007; 146: 975-979.

25. LaWrence D, Mitrou F, Zubrick SR. Smoking and mental illness: results from population surveys in Australia and the United States. BMC Public Health 2009; 9: 285.

26. Zikos E, Gill KJ, ChaRney DA. Personality disorders among alcoholic outpatients: prevalence and course in treatment. Can J Psychiatry 2010; 55: 65-73.

27. Fydrich T, Ulsmann D. Comorbidity of chronic somatic illness and psychological disorders. Problems in outpatient mental health care. Bundesgesundheitsblatt Gesundheitsforschung Gesundheitsschutz 2011; 54: 108-119.

28. Esteban y Peña MM, Hernández Barrera V, FernÁndez Cordero X, Gil de Miguel A, Rodríguez PéREZ M, LÓPEZ-DE ANDREs A et al. Self-perception of health status, mental health and quality of life among adults with diabetes residing in a metropolitan area. Diabetes Metab 2010; 36: 305-311.

29. Berglund B; Lindvall T, Schwela D, WHO. Community Noise. Disponible en: http://www. bvsde. paho. org/ bvsci/i/fulltext/noise/noise.pdf. Consultado en octubre 2011.

30. Gabe J, Williams P. Women, housing, and mental health. Int J Health Serv 1987; 17: 667-679. 